Gleichberechtigung

\section{Salto vorwärts oder rückwärts?}

Corona, so derzeit die Hoffnung mancher, könnte neben all den unerfreulichen Konsequenzen möglicherweise immerhin eines schaffen, nämlich die Arbeitsteilung in Paarhaushalten etwas mehr in Richtung Gleichverteilung verschieben. Drei Wirkungskanäle lassen sich in der Debatte identifizieren. Erstens wird eine Aufwertung von systemrelevanten Berufen erwartet, von denen ein Gutteil frauendominiert ist. Der positive Effekt auf die Frauenlöhne senkt den Gender Pay Gap, sodass der komparative Vorteil von Frauen für Erwerbsarbeit steigt und sich zudem ihre Verhandlungsposition im Paar verbessert. Zweitens wird davon ausgegangen, dass Corona einen Digitalisierungsschub bringt. Unumkehrbare Lerneffekte bezüglich der Machbarkeit und Produktivität von Homeoffice werden Präsenzkulturen weiter zurückdrängen. Flexible Arbeit und Telearbeit werden keine Karrierekiller mehr sein. Der dritte Wirkungsmechanismus läuft über die Erwartung, dass in Corona-Zeiten Homeoffice-fähige Väter mehr Zeit für Kinderbetreuung aufwenden. Dadurch werden neue Rollen eingeübt, die zu einer nachhaltigen Entlastung von Frauen und Müttern durch unbezahlte Arbeit führen. Diese könnten dann mehr Zeit und Energie für ihre berufliche Entwicklung aufwenden. Ach wie schön wäre es doch, wenn es dazu käme. Wie erfreulich wäre es, wenn wir in zehn Jahren rückblickend mit Corona nicht nur einen positiven Klimaeffekt, sondern auch einen Sprung vorwärts in Sachen Gleichstellung verbänden!

Die Befunde deuten derzeit leider eher in die gegenteilige Richtung. Wie Beschäftigtenbefragungen in der Krise zeigen, sind es derzeit die Frauen, die den überwiegenden Teil der Betreuungsarbeit übernehmen, wenn Kinder im Haushalt zu versorgen sind. Mütter schränken in diesen Tagen häufiger ihre Arbeitszeit ein und gehen sogar häufiger als Väter gar keiner bezahlten Tätigkeit nach, wie andere Umfragen verdeutlichen. Wirklich überraschend ist dies nicht. Ein Anpassungsdruck in Richtung eines „Mehr“ an väterlicher Kinderbetreuung kommt nicht automatisch durch erhöhte Präsenzzeiten des Vaters zuhause zustande. Grundsätzlich entsteht ein Anpassungsdruck über ein verändertes relatives Zeitbudget der Partner nur dann, wenn diese Veränderung nicht durch eine ungünstige Veränderung des Einkommensverhältnisses der Partner oder gegenläufig wirkende Geschlechterrollen ausgehebelt wird. Die Coro-

(c) Der/die Autor(en) 2020. Open Access: Dieser Artikel wird unter der Creative Commons Namensnennung 4.0 International Lizenz (https:// creativecommons.org/licenses/by/4.0/deed.de) veröffentlicht.

Open Access wird durch die ZBW - Leibniz-Informationszentrum Wirtschaft gefördert.
na-Krise entfaltet hier leider mehrere Wirkungen zugleich, und zwar in unterschiedliche Richtungen.

Personen, die im Homeoffice arbeiten, sind eher höher gebildet, arbeiten seltener in Branchen, die in der Krise Kurzarbeit einsetzen. Sie haben, soweit sie nicht selbstständig beschäftigt sind, derzeit ein geringeres Job- und Einkommensrisiko. Ein akademisch gebildeter Vater im Homeoffice bringt daher zwar zunächst ein zusätzliches Zeitbudget ein; dies ändert sich jedoch, wenn der Frau als Selbstständige die Aufträge fehlen oder ihr Betrieb Kurzarbeit angemeldet hat. Dann hat auch sie mehr Zeit und mit dem Einkommensverhältnis hat sich auch der komparative Vorteil für Erwerbsarbeit und die relative Verhandlungsposition im Paar zuungunsten der Frau verschoben. Trifft die Arbeitslosigkeit hingegen inn, ist, wo Männlichkeitskonzepte an die Ernährerrolle gekoppelt sind, ebenfalls nicht unbedingt mit einem gleichstellungspolitischen Fortschritt zu rechnen: Arbeitslose Männer engagieren sich deutlich weniger im Haushalt als arbeitslose Frauen. Und selbst wenn sie es ist, die den sicheren Job hat: Sie verdient pro Stunde durchschnittlich $20 \%$ weniger als er, sodass die Haushaltskasse ihre Arbeitszeiteinschränkung besser verkraftet als seine.

Wenn Frauen in der Krise ohne Einkommen dastehen, liegt das oft auch daran, dass sie die Mehrheit unter den Minijobber*innen stellen, die gerade in krisengeplagten Branchen wie Handel und Gastgewerbe eine große Rolle spielen. Geringfügig Beschäftigte haben keinen Anspruch auf gesetzliches Kurzarbeitergeld. Da Frauen häufiger in kleinen Betrieben ohne Tarifvertrag arbeiten, wird das Kurzarbeitergeld zudem auch seltener aufgestockt. Und die Steuerklasse 5 stellt Frauen durch die Nettoentgeltbindung des Kurzarbeitergelds in Deutschland ein zusätzliches Bein. Hinzu kommt, dass Frauen derzeit auch häufiger (unbezahlt) freigestellt sind als Männer. Neben strukturellen Ungleichheiten der Geschlechter tragen nach wie vor verankerte traditionelle Rollenbilder dazu bei, dass der Löwenanteil der unbezahlten Arbeit, die in Corona-Zeiten neben der Hausarbeit und der Betreuung von Kindern auch deren Beschulung umfasst, an den Frauen hängen bleibt. Schon vor Corona galt dies auch für Paare in Vollzeit-Vollzeit-Konstellationen. Es ist derzeit nicht ausgemacht, wie das Corona-Experiment ausgeht: Ob wir einen Salto vorwärts oder rückwärts in Sachen Gleichberechtigung machen. Ob sich die Ungleichheit in der Krise reduziert oder reproduziert, wird davon abhängen, was Betriebe, Sozialpartner und Staat sowie wir alle aus dieser Krise lernen.

Christina Boll

Deutsches Jugendinstitut boll@dji.de 